\title{
MORPHOLOGY OF HYDROGEN-BONDING POLYMER COMPLEX AGGREGATES FORMED FROM DILUTE SOLUTIONS BY FREEZE-DEFREEZE METHOD
}

\author{
Hae Jinn Kim and Munenori Sakamoto \\ Faculty of Engineering, Tokyo Institute or Technology \\ O-okayama, Meguro-ku, Tokyo, 152 Japan
}

\begin{abstract}
Morphology of aggregates separated from dilute aqueous solutions of hydrogen-bonding polymer comlex systems by rapid freezing at $-78^{\circ} \mathrm{C}$ followed by defreezing was studied by scanning elec tron microscopy. Polymer complexes with weak cooperative interaction gave no precipitates and those with moderate interaction yielded aggregates in fibrous form. Polymer complexes with strong interaction gave aggregates of network structure which was probably formed from the modulated structure developed by spinodal decomposition during the rapid freezing treatment of the solution.
\end{abstract}

Hydrogen-bond donor polymers and hydrogen-bond acceptor polymers often form polymer-complex molecules in aqueous solutions. The polymer-complex molecules aggregate spontaneously or aggregate only when the solution parameters such as temperature and concentration are changed favorably. Both cooperative hydrogen bonding and concerted hydrophobic interaction between the two polymer species are responsible for the complex formation and the aggregation.

We reported in the preceding paper [1] that fib. rous aggregates were obtained in a good yield when a dilute solution of polymer complex (PC) of stpoly(methacrylic acid) (st-PMAA) and poly(ethylene oxide) (PEO) was frozen rapidly and the frozen solu. tion was defrozen. Such fibrous aggregates were often reported for polyion complex systems. For example, Tsuchida et al. [2] reported that fibrous aggregates were separated, very slowly on standing, from a dilute solution of PC of at-PMAA and a rigid polycation. Compared to such spontaneous slow aggregation of polyion complex systems via nucleation and growth mechanism, the aggregation of PC of st-PMAA and PEO through the freeze-defreeze (FD) method is a rapid process. It is of some value to extend the FD method to varieties of hydrogen-bonding PC systems of various stabilities for the preparation of aggregates of ordered morphology.

Hydrogen-bond donor polymers studied were at- poly(acrylic acid) (PAA) of $\mathrm{MW}$ of $330 \times 10^{3}$, atPMAA of MW of $180 \times 10^{3}$ and triad syndiotacticity (S) of $57 \%$, and $s t$-PMAA of MW of $230 \times 10^{3}$ and S of $92 \%$. Hydrogen-bond acceptor polymers studied were $a t$-poly(vinyl alcohol) (PVA) of $\mathrm{MW}$ of $880 \times$ $10^{3}, \mathrm{PEO}$ of different $\mathrm{MW}\left(\mathrm{PEO}_{\mathrm{L}}: 6 \times 10^{3}\right.$ and $\mathrm{PEO}_{\mathrm{H}}$ : $\left.20 \times 10^{3}\right)$, poly-at-( $N$-vinylpyrrolidone) (PVP) of $\mathrm{MW}$ of $360 \times 10^{3}$, and at-poly(acrylamide) (PAAm) of MW of $120 \times 10^{3}$.

Equal volume of 0.25 (unit mmol)/L aqueous solutions of a hydrogen-bond donor polymer and an acceptor polymer were mixed at room temperature with stirring. The mixed clear solution was placed in a test tube (i. d., $10 \mathrm{~mm}$ ) and the tube was put into a bath at $-78^{\circ} \mathrm{C}$. The tube was removed from the bath and the frozen mixture was allowed to melt at room temperature. In most cases, a suspension was obtained, from which PC aggregates were precipitated. The products were separated by centrifugation and washed with water several times. The dried precipitates were spattered with $\mathrm{Au}$ and the morphology was studied by scanning electron microscopy (SEM) with a JEOL scanning electron micrograph, JSM-200.

From solutions of PCs of PVA, no aggregates were separated by the FD technique. No precipitates were also formed from PC of PAA and PEO. Precipitates of disordered structure were obtained from at-PMAA and PEO [1]. From solutions of st-PMAA and PEO, fibrous aggregates were precipitated. The morphology 


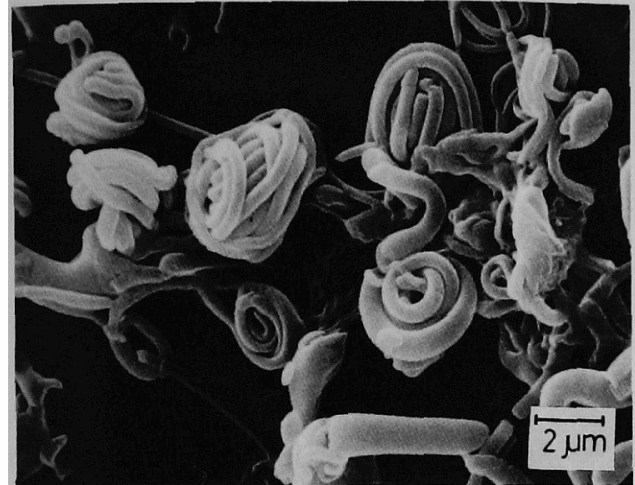

Fig. 1. SEM photograph of aggregates of PC of stPMAA and $\mathrm{PEO}_{\mathrm{L}}$.

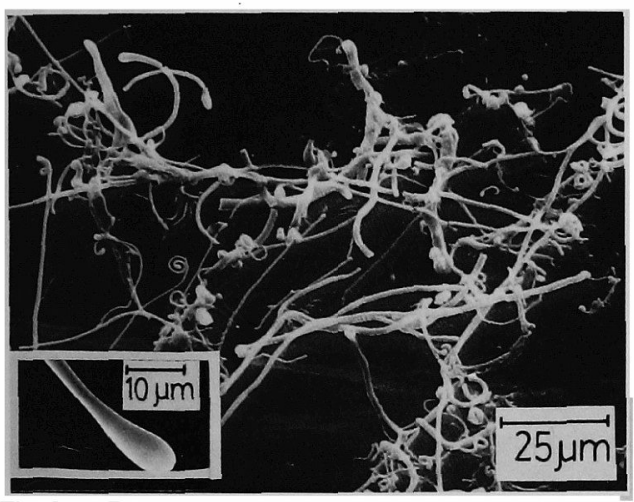

Fig. 2. SEM photograph of aggregates of PC of stPMAA and $\mathrm{PEO}_{\mathrm{H}}$.

of the fibrous aggregates depended on the $\mathrm{MW}$ of PEO [1]. Fig. 1. shows the aggregates of PC of stPMAA and $\mathrm{PEO}_{\mathrm{L}}$. A typical precipitate is composed of a long flexible fiber of a relatively uniform diameter (ca. $0.5 \mu \mathrm{m}$ ) which coils up to form a globule of a diameter of ca. $3 \mu \mathrm{m}$. Fig. 2 shows the aggregates of $\mathrm{PC}$ of at-PMAA and $\mathrm{PEO}_{\mathrm{H}}$. Long, flexible fibers of different diameters are entangled in this case. In the present study, we realized that some of the fiber ends have a droplet shape (see the inserted picture in Fig. 2).

Figs. 3 and 4 show fibrous aggreagtes formed from PC of PAA and PVP. Fibers with a diameter of ca. 1 $\mu \mathrm{m}$ are entangled. The fibers seem more rigid than those from st-PMAA and PEOs. Fig. 5 shows aggregates formed from PC of st-PMAA and PVP. Similar aggregates are formed from PC of $a t$-PMAA and PVP. A typical aggregate is a short, straight fiber. Some of

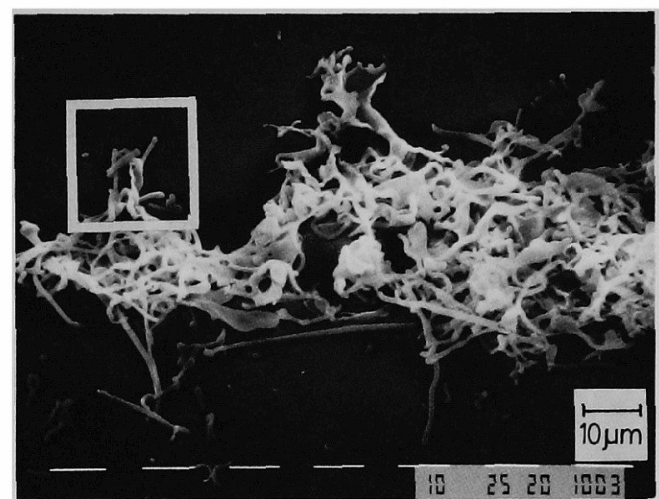

Fig. 3. SEM photograph of aggregates of $P C$ of PAA and PVP.

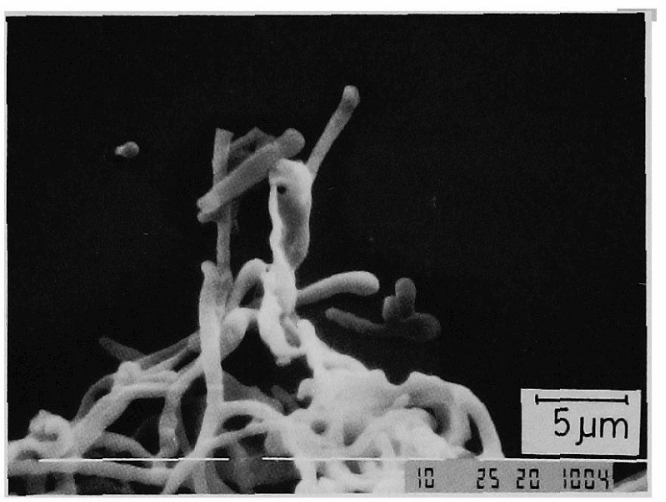

Fig. 4. The blocked area in Fig. 3 at a higher magnification.

the fiber ends have a droplet shape. Another type of the fiber ends looks like a cylinder bottom as if a rigid rod is cut perpendicularly. Some of the fibers have Y-shaped branches as shown in Fig. 6.

The morphology of aggregates formed from PC of PAA and PAAm shown in Fig. 7 is quite different from those already shown. The precipitates have a network structure with irregularly shaped fibers. The unique continuous network structure, or interconnecting lacy structure [3], of aggregates is more evident in Fig. 8 that shows aggregates formed from PC of atPMAA and PAAm. A similar structure was observed for the aggregates formed from PC of st-PMAA and PAAm.

All the aggregates obtained in this study were amorphous and no molecular orientation was observed for these samples.

The results obtained in this study suggest that the 


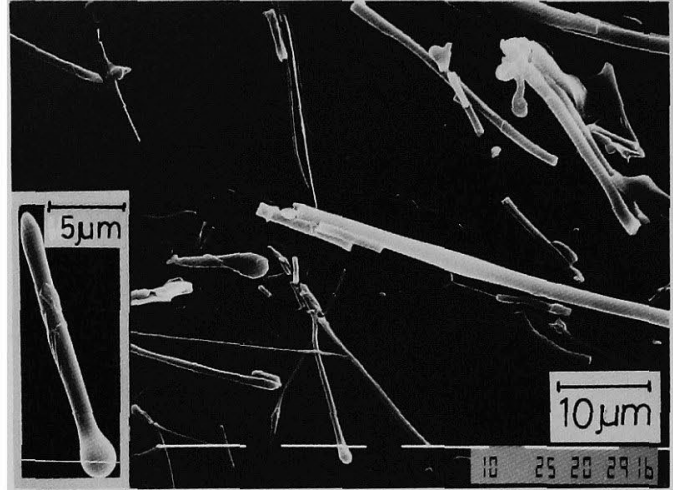

Fig. 5. SEM photograph of aggregates of PC of $s t$ PMAA and PVP.

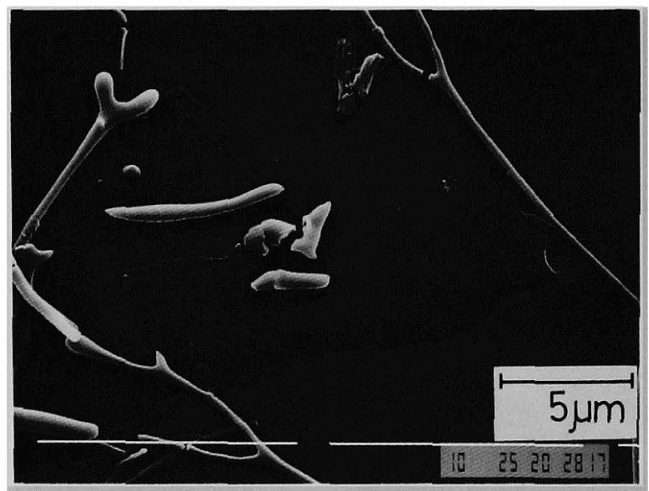

Fig. 6. SEM photograph of aggregates of PAA and PVP.

morphology of the aggregates of PC systems is related with the strength of the cooperative interaction in the PC or the stability of the PC.

The stability of PCs of polyacids with PEO was reported to increase in the order $[4,5]$ :

PAA $<a t$-PMAA $<s t$-PMA

The stability of the PCs of hydrogen-bond acceptor polymers with at-PMAA is considered to increase in the order [4]:

$$
\text { PVA }<\text { PEO }<\text { PVP }<\text { PAAm }
$$

The molecular weight of polymer is also an important factor. PEO of higher molecular weight gave a more stable PC than PEO of lower molecular weight [4].

With an assumption that the order of the cooperative interaction given above be applicable to any combination between hydrogen-bond donor polymers and acceptor polymers, we can summarize the morphological obvervation in this study as follows: weak PCs

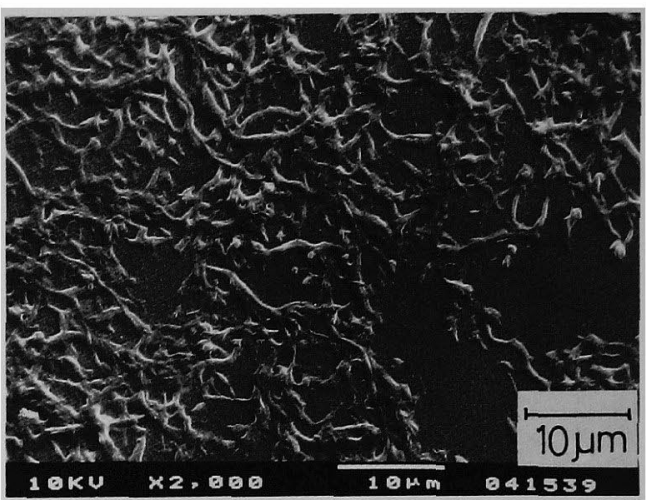

Fig. 7. SEM photograph of aggregates of PAA and PAAm.

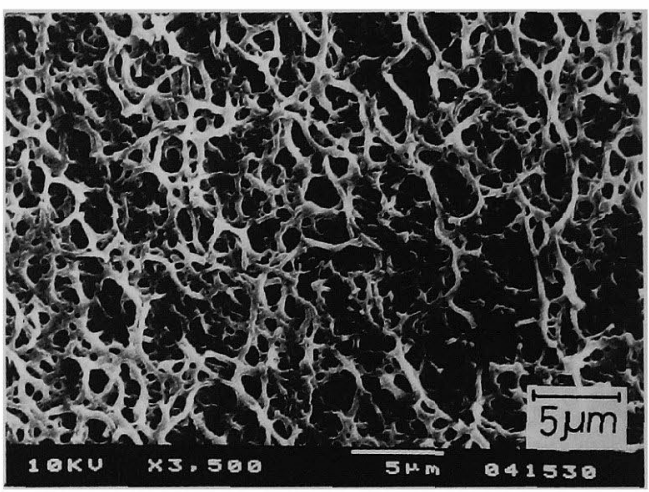

Fig. 8. SEM photograph of aggregates of st-PMAA and PAAm.

such as PC of PAA and PVA do not form any aggregates by the FD technique. Less weak $P C$ of atPMAA and PEO forms precipitates without ordered structure. PCs of moderate interaction give fibrous aggregates by the FD technique. Less stable PCs in this category seem to yield more flexible fibers.

The strong PCs such as PC of at-PMAA and PAAm give a continuous network structure. The network structure in Fig. 8 is similar to that observed for a film made by drying a hydrogel membrane in modulated structure $[3,6]$. The modulated structure was formed via spinodal decomposition [7] during hy. drogel formation.

It is likely that spinodal decomposition takes place when a dilute solution of PC of strong cooperative interaction is cooled rapidly in freezing. Then, the aggregates structure must reflect the modulated structure formed in the course of spinodal decomposition. 
When the frozen mixture was defrozen in this case, the defrozen state was a semisolid rather than a sus. pension. The semisolid structure was gradually broken and a soft, bulky, cotton-ball-like precipitate was formed. This fact also suggests the formation of the modulated structure during the freezing.

It is uncertain at present whether those fibrous aggregates from $\mathrm{PCs}$ of moderate interaction were also formed through spinodal decomposition. The modulated structure, if formed, could be much deformed during defreezing, collecting, drying, or SEM sample preparation.

In many cases, globular aggregates such as seen in Figs. 4 and 6 are present in the precipitates. When the solution was frozen at a slower rate at a higher temperature (e. g., $-20^{\circ} \mathrm{C}$ ), the main form of the precipitates was globular. The globular aggregates formed at $-78^{\circ} \mathrm{C}$ seem to be contaminates produced at the inner part of the test tube where cooling rate must be lower. This consideration is in favor of spi- nodal decomposition mechanism: a rapid change in temperature or composition from the homogeneous state to the heterogeneous spinodal region is essential for spinodal decomposition to take place.

\section{REFERENCES}

1. H. J. Kim, M. Sakamoto, and H. Tonami, Kobunshi Ronbunshu, 40, 579 (1983)

2. E. Tsuchida, K. Abe, and M. Honma, Macromolecules, 9, 112 (1976)

3. P. J. Tsai and J. M. Torkelson, Macromolecules, 23, 775 (1990)

4. E. Tsuchida and K. Abe, Adv. Polym. Sci., 45, 1 (1982)

5. H. J. Kim, K. Mogami, M. Sakamoto, and H. Tonami, Kobunshi Ronbunshu, 40, 637 (1983)

6. H. J. Kim, K. Mimura, T. Inoue, and M. Sakamoto, Polym. Prepr., Jpn., 34, 1648 (1985)

7. T. Inoue, T. Ougizawa, O. Yasuda, and K. Miyasaka, Macromolecules, 18, 57 (1985) 